\title{
The hot rim sign on hepatobiliary scintigraphy (HIDA) with CT correlation
}

\author{
Ian Blake Amber, ${ }^{1}$ Joshua Leighton, ${ }^{1}$ Su-Yu Li, ${ }^{1}$ Gary Stuart Greene ${ }^{2}$ \\ ${ }^{1}$ Radiology Department, The Pennsylvania Hospital, University of Penn Health System, Philadelphia, Pennsylvania, USA; \\ ${ }^{2}$ Radiology/Nuclear Medicine Department, The Pennsylvania Hospital, University of Penn Health System, Philadelphia, Pennsylvania, USA
}

Correspondence to Dr Gary Stuart Greene, greeneg@pahosp.com

\section{Summary}

An older male with multiple medical comorbidities presented to the emergency room after 3 days of worsening right upper quadrant pain. The patient had an elevated white blood cell count and mildly elevated liver functions. Initial ultrasound was equivocal and further imaging with CT scan was obtained. The CT scan was read as suggestive of cholecystitis, however a hepatobiliary scintigraphy (HIDA) scan was ordered for confirmation, as the patient was a poor operative candidate. The HIDA demonstrated no bile duct or small bowel activity on initial images or delays, however a classic 'hot rim' sign was present, confirming acute cholecystitis. The patient ultimately underwent percutaneous cholecystostomy with drainage for treatment where acute cholecystitis was confirmed. Upon retrospective review, the CT demonstrated hyperaemia surrounding the gallbladder fossa, which is the CT scan equivalent of a scintigraphic 'hot rim' sign. This is an uncommon example of a radiologic sign correlation between multiple imaging modalities.

\section{BACKGROUND}

Patients presenting with right upper quadrant pain often have gallbladder or biliary dysfunction. The standard of care with regard to imaging is to begin the investigation with sonographic evaluation of the abdomen. When there are equivocal ultrasonographic findings, and further imaging investigation is needed, hepatobiliary scintigraphy (HIDA) is typically the next step as it has superior specificity, however, CT is now commonly performed as the next step if the patient is unstable or if there is more diagnostic uncertainty as to the etiology of the patient's symptoms. ${ }^{1}$

If a HIDA scan is performed, there is intravenous injection of Technetium-99 m labelled radiotracer, typically following by prompt hepatic uptake. ${ }^{1}$ If there is biliary excretion and the gallbladder fails to uptake tracer within $1 \mathrm{~h}$, the diagnosis of acute cholecystitis is made and the appropriate clinical action can be taken. A secondary finding of acute cholecystitis, associated with a non-visualised

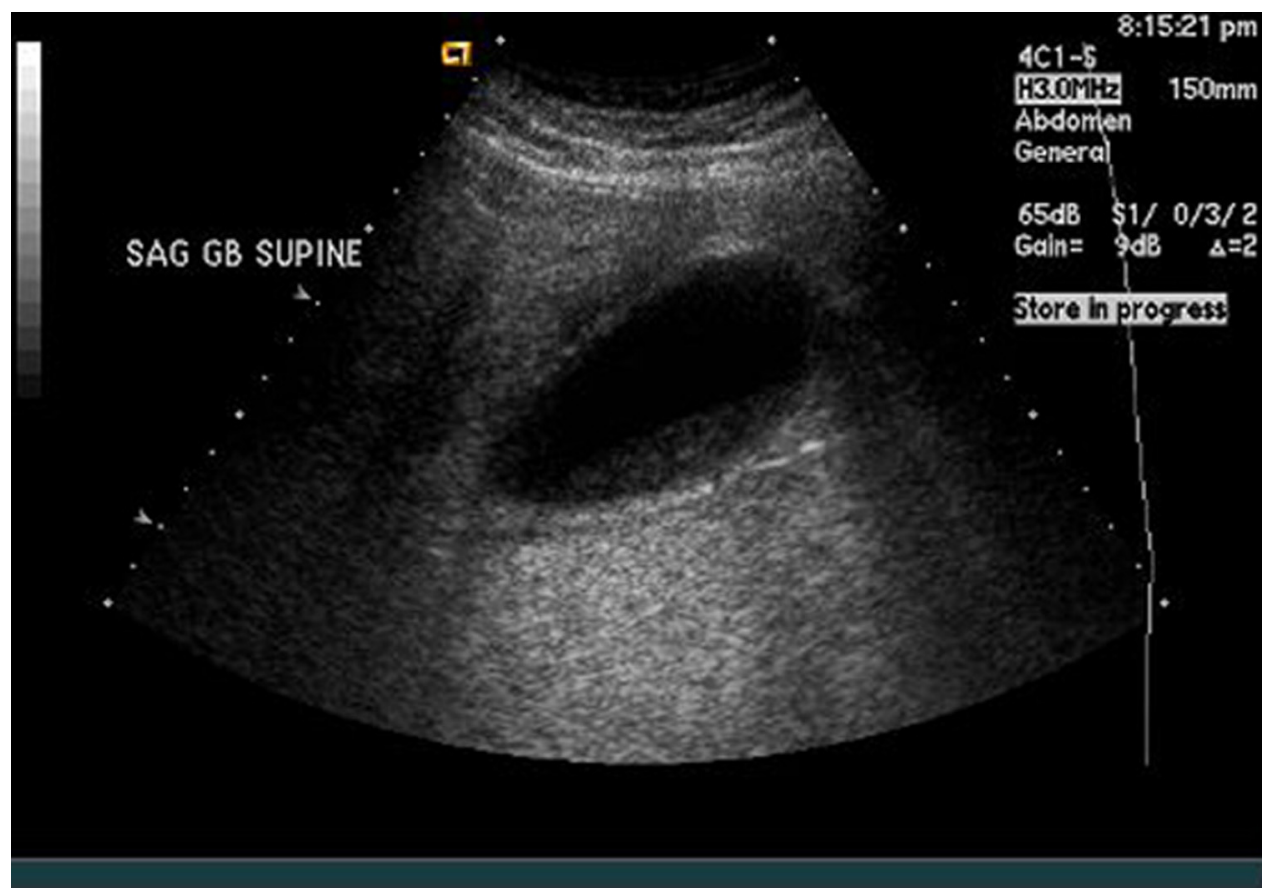

Figure 1 Initial abdominal ultrasound image in the sagittal plane, showing sludge in the patient's gallbladder without evidence of cholelithiasis. 


\section{BMJ Case Reports}

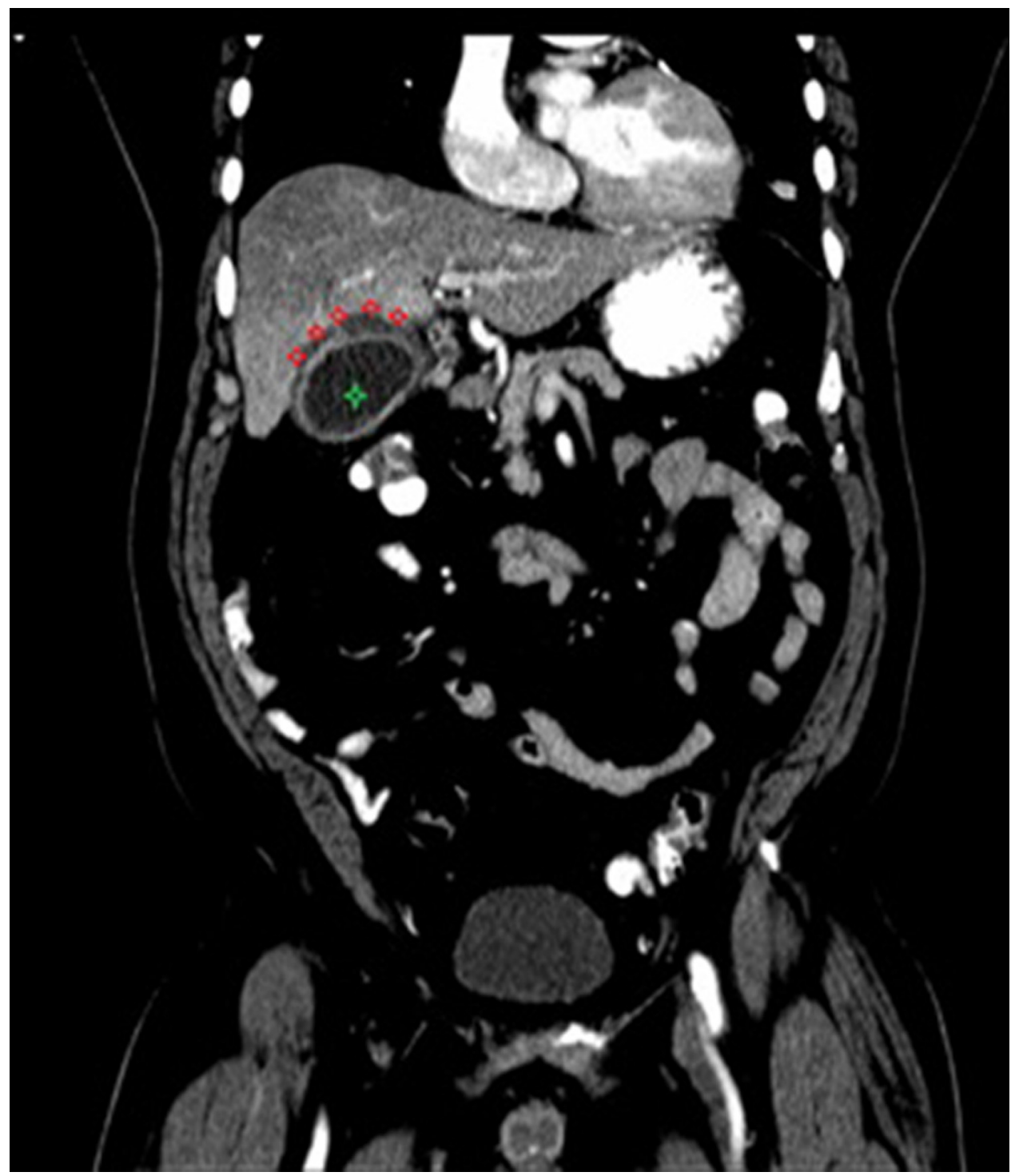

Figure 2 A coronal view of the CT abdomen and pelvis. The green star is within the gallbladder. Note the sludge inside the gallbladder, as well as the thickened wall and surrounding pericholecystic fluid. The red diamonds outline the reactive hepatic parenchymal tissue, creating the 'hot rim' sign on the CT scan.

gallbladder is known as the 'hot rim' sign. ${ }^{2}$ It is defined as a peripherally enhancing rim surrounding a photopenic gallbladder fossa. ${ }^{1-3}$

The most commonly accepted pathophysiologic mechanism for the appearance of the 'hot rim' sign is inflammatory changes from the gallbladder spreading to and affecting the surrounding liver. ${ }^{13}$ The 'hot rim' sign has clinical relevance, because it is associated with a high incidence of perforated or gangrenous cholecystitis. ${ }^{3}$ The presence of these aforementioned conditions increases the likelihood of complications, and warrants urgent surgical evaluation.

While a HIDA scan is the best test for evaluating acute cholecystitis, it is time consuming. In patients who are acutely ill, often times the treating team will opt for a CT scan first. The traditional CT scan findings associated with acute cholecystitis are: gallstones, gallbladder distention and wall thickening and stranding of pericholecystic fat. ${ }^{4}$ Additionally, there is a CT scan equivalent of the "hot rim' sign, which presents as a crescent shaped region of increased uptake within the hepatic parenchyma adjacent to the inflamed gall bladder. ${ }^{5}$ While the significance of a rim sign on CT scan has not been fully evaluated, it is important to recognise that given the increased morbidity associated with a gangrenous gall bladder, the patient requires the same urgent level of treatment as if the findings were seen on a HIDA scan.

\section{CASE PRESENTATION}

An older male presented to the emergency room (ER) after being sent by his primary care physician (PCP) for abdominal and chest pain. The patient had 3 days of predominantly right upper quadrant abdominal pain, which then began to radiate to both his chest and peri-umbilical region. The pain continued to increase in intensity, and he subsequently left work to see his PCP. On further questioning, he felt nausea, a decrease in appetite and chills. He denied any relieving factors. Specifically, the pain did not 


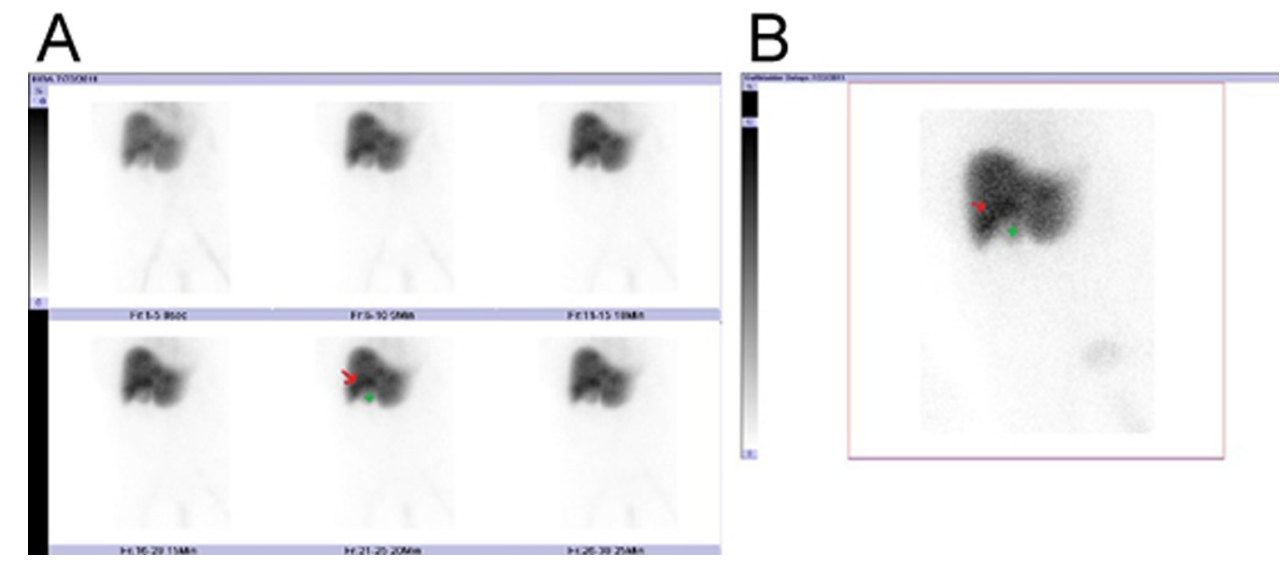

Figure 3 (A) Images taken from the patient's hepatobiliary scintigraphy (HIDA) scan. The green star is located in the photopenic gallbladder fossa. The red arrow points to a surrounding crescent shaped area of increased uptake- the 'hot rim' sign. (B) Delayed image from the patient's HIDA scan. The green star is located in the photopenic gallbladder fossa. The red arrow points to a surrounding crescent shaped area of increased uptake- the 'hot rim' sign. Note that there is no evidence of uptake within the duodenum, signifying biliary obstruction.

change with food or positioning. He was sent directly by his PCP to the ER.

The patient has a medical history of heartburn, diabetes, hypertension, hyperlipidemia and coronary artery disease. The patient had prior coronary artery bypass grafting, as well as bilateral knee replacements. The patient denied smoking currently, but has smoked a total of 12 pack years previously. He last used tobacco in 1963. He denied drug and alcohol use. He is a widower, and lives on his own.

\section{INVESTIGATIONS}

After history and physical examination, laboratory work was ordered. The pertinent positives were a white bllod cell count of 19.3 and aspartate aminotransferase/alanine aminotransferase/alkaline phosphatase of 75/83/194 respectively. An ultrasound was performed, which showed gallbladder sludge, wall thickening and a suggestion of pericholecystic fluid (figure 1). Of note there were no gallstones or sonographic Murphy's sign.

Equivocal ultrasound findings necessitated further imaging. Due to the patient's clinical status, a CT scan of the abdomen and pelvis was ordered. This showed a moderately distended gallbladder with moderate wall thickening and mucosal hyperenhancement (figure 2). No gallstones were visualised.

Given the patient's multiple comorbidities, both surgery and percutaneous cholecystostomy were considered high risk. Therefore, further confirmatory diagnostic imaging was required. A HIDA scan was performed, which showed a hot rim sign, with no evidence of tracer expulsion into the duodenum (figure 3). This indicated that there was most likely a complicated case of cholecystitis as well as blockage of the common bile duct. The blockage of the bile duct was likely secondary to the underlying inflammation surrounding the gallbladder. There was no evidence of biliary obstruction on further imaging.

\section{DIFFERENTIAL DIAGNOSIS}

Acute cholecystitis, acalculous cholecystitis and acute cholecystitis with high-grade extra-hepatic bile duct obstruction.

\section{TREATMENT}

With the diagnosis of acute cholecystitis, the patient required treatment. Given the patient's multiple risk factors, a percutaneous cholecystostomy was performed. Fluid was drained and cultured.

\section{OUTCOME AND FOLLOW-UP}

Following drainage, the patient's clinical status improved significantly. Cultures from the fluid demonstrated abundant Klebsiella. The patient was placed on an antibiotic regimen of levoquin and flagyl. A cholecystomy tube was to remain in place until cleared medically for surgery. However, before being able to return for surgery, the patient succumbed to complications from his illness.

\section{DISCUSSION}

The 'hot rim' sign has been an established marker in HIDA since it was first reported in $1984 .{ }^{6}$ It is typically seen as a secondary, albeit more specific finding of cholecystitis. It is also a marker of disease severity, as it has an association with more severe forms of cholecystitis, such as perforated and gangrenous cholecystitis. ${ }^{3}$ Typically, acute cholecystitis is heralded on HIDA as non-visualisation of the gallbladder after uptake within the biliary tree. However, the 'hot rim sign' is independent of biliary excretion and instead depends upon the inflammatory reaction of the hepatic parenchyma adjacent to the gallbladder fossa and the subsequently resulting hyperemia. ${ }^{6}$

The above mentioned pathophysiologic mechanism of the 'hot rim' sign proved useful in diagnosing our patient, as one of our differential considerations prior to additional imaging, was acalculous cholecystitis. The 'hot rim' sign lacks association with cases of acalculous cholecystitis, likely secondary to its pathophysiologic cause. Acalculous cholecystitis is typically due to gallbladder immotility in a critically ill patient, with subsequent ischaemic insult secondary to the underlying toxic exposure of bile. ${ }^{7}$ During the early phase of this process, there is not enough of a parenchymal inflammatory response to generate a 'hot rim' sign. Only in prolonged cases of acalculous cholecystitis where the gallbladder necroses or perforates would a 


\section{BMJ Case Reports}

sign be generated. ${ }^{7}$ Therefore, after the HIDA scan, we felt it was less likely to be a rare case of acalculous cholecystitis and more likely to be acute cholecystitis.

As CT imaging has become widespread in use, it is slowly supplanting the HIDA scan as the next step in evaluating a more acutely ill patient with suspected acute cholecystitis and an equivocal ultrasound. It is therefore important to be aware of the presence of the CT scan correlate of the "hot rim' sign, appreciate the mechanisms which cause it, and understand the subsequent steps in patient management.

\section{Learning points}

- A hot rim sign is a peripherally enhancing rim surrounding a photopenic gallbladder fossa demonstrated on a HIDA scan.

- The hot rim sign is caused by radiotracer uptake of surrounding inflammatory parenchyma.

- The CT scan equivalent of a scintigraphic hot rim sign is crescent shaped liver enhancement surrounding an inflamed gallbladder.

- A hot rim sign is associated with an increased incidence of gangrenous and/or perforated gall bladder.

- If a hot rim sign is seen on either CT scan or HIDA, urgent surgical evaluation is needed.
Competing interests None.

Patient consent Not obtained.

\section{REFERENCES}

1. Ziessman HA. Acute cholecystitis, biliary obstruction, and biliary leakage. Semin Nucl Med 2003;33:279-96.

2. Joseph UA, Barron BJ, Lamki LM. Rim sign in Tc-99m sulfur colloid hepatic scintigraphy. Clin Nucl Med 2005:30:284-5.

3. Swayne LC, Filippone A. Gallbladder perforation: correlation of cholescintigraphic and sonographic findings with the Niemeier classification. $J$ Nucl Med 1990;31:1915-20.

4. Paulson EK. Acute cholecystitis: CT findings. Semin Ultrasound CT MR 2000;21:56-63.

5. Yamashita K, Jin MJ, Hirose Y, et al. CT finding of transient focal increased attenuation of the liver adjacent to the gallbladder in acute cholecystitis. AJR Am J Roentgenol 1995;164:343-6.

6. Cawthon MA, Brown DM, Hartshorne MF, et al. Biliary scintigraphy. The "hot rim" sign. Clin Nucl Med 1984;9:619-21.

7. Owen CC, Jain R. Acute Acalculous Cholecystitis. Curr Treat Options Gastroenterol 2005;8:99-104.

This pdf has been created automatically from the final edited text and images.

Copyright 2012 BMJ Publishing Group. All rights reserved. For permission to reuse any of this content visit http://group.bmj.com/group/rights-licensing/permissions.

BMJ Case Report Fellows may re-use this article for personal use and teaching without any further permission.

Please cite this article as follows (you will need to access the article online to obtain the date of publication).

Amber IB, Leighton J, Li SY, Greene GS. The hot rim sign on hepatobiliary scintigraphy (HIDA) with CT correlation. BMJ Case Reports 2012; 10.1136/bcr.09.2011.4778, Published XXX

Become a Fellow of BMJ Case Reports today and you can:

- Submit as many cases as you like

- Enjoy fast sympathetic peer review and rapid publication of accepted articles

- Access all the published articles

- Re-use any of the published material for personal use and teaching without further permission

For information on Institutional Fellowships contact consortiasales@bmjgroup.com

Visit casereports.bmj.com for more articles like this and to become a Fellow 